\title{
On certain subclasses of analytic functions associated with Poisson distribution series
}

\author{
B. A. Frasin \\ Department of Mathematics, \\ Al al-Bayt University, Mafraq, Jordan \\ email: bafrasin@yahoo.com
}

\begin{abstract}
In this paper, we find the necessary and sufficient conditions, inclusion relations for Poisson distribution series $\mathcal{K}(\mathrm{m}, z)=z+$ $\sum_{n=2}^{\infty} \frac{m^{n-1}}{(n-1) !} e^{-m} z^{n}$ to be in the subclasses $\mathcal{S}(k, \lambda)$ and $\mathcal{C}(k, \lambda)$ of analytic functions with negative coefficients. Further, we obtain necessary and sufficient conditions for the integral operator $\mathcal{G}(m, z)=\int_{0}^{z} \frac{\mathcal{F}(m, t)}{t} d t$ to be in the above classes.
\end{abstract}

\section{Introduction and definitions}

Let $\mathcal{A}$ denote the class of the normalized functions of the form

$$
f(z)=z+\sum_{n=2}^{\infty} a_{n} z^{n}
$$

which are analytic in the open unit disk $\mathcal{U}=\{z \in \mathbb{C}:|z|<1\}$. Further, let $\mathcal{T}$ be a subclass of $\mathcal{A}$ consisting of functions of the form,

$$
f(z)=z-\sum_{n=2}^{\infty}\left|a_{n}\right| z^{n}, \quad z \in \mathcal{U}
$$


A function $f$ of the form $(2)$ is in $\mathcal{S}(k, \lambda)$ if it satisfies the condition

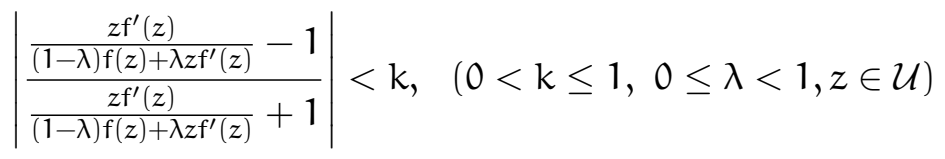

and $f \in \mathcal{C}(k, \lambda)$ if and only if $z f^{\prime} \in \mathcal{S}(k, \lambda)$. The class $\mathcal{S}(k, \lambda)$ was introduced by Frasin et al. [3].

We note that $\mathcal{S}(\mathrm{k}, 0)=\mathcal{S}(\mathrm{k})$ and $\mathcal{C}(\mathrm{k}, 0)=\mathcal{C}(\mathrm{k})$, where the classes $\mathcal{S}(\mathrm{k})$ and $\mathcal{C}(k)$ were introduced and studied by Padmanabhan [9] (see also, [5], [8]).

A function $\mathrm{f} \in \mathcal{A}$ is said to be in the class $\mathcal{R}^{\tau}(\mathrm{A}, \mathrm{B}), \tau \in \mathbb{C} \backslash\{0\},-1 \leq \mathrm{B}<$ $A \leq 1$, if it satisfies the inequality

$$
\left|\frac{f^{\prime}(z)-1}{(A-B) \tau-B\left[f^{\prime}(z)-1\right]}\right|<1, \quad z \in \mathcal{U} .
$$

This class was introduced by Dixit and Pal [2].

A variable $x$ is said to be Poisson distributed if it takes the values $0,1,2,3, \ldots$ with probabilities $e^{-m}, m \frac{e^{-m}}{1 !}, m^{2} \frac{e^{-m}}{2 !}, m^{3} \frac{e^{-m}}{3 !}, \ldots$ respectively, where $m$ is called the parameter. Thus

$$
P(x=r)=\frac{m^{r} e^{-m}}{r !}, r=0,1,2,3, \ldots
$$

Very recently, Porwal [10] (see also, [6, 7]) introduce a power series whose coefficients are probabilities of Poisson distribution

$$
\mathcal{K}(\mathrm{m}, z)=z+\sum_{n=2}^{\infty} \frac{m^{n-1}}{(n-1) !} e^{-m} z^{n}, \quad z \in \mathcal{U},
$$

where $m>0$. By ratio test the radius of convergence of above series is infinity. In [10], Porwal also defined the series

$$
\mathcal{F}(m, z)=2 z-\mathcal{K}(m, z)=z-\sum_{n=2}^{\infty} \frac{m^{n-1}}{(n-1) !} e^{-m} z^{n}, \quad z \in \mathcal{U} .
$$

Using the Hadamard product, Porwal and Kumar [12] introduced a new linear operator $\mathcal{I}(\mathrm{m}, z): \mathcal{A} \rightarrow \mathcal{A}$ defined by

$$
\mathcal{I}(\mathrm{m}, z) \mathrm{f}=\mathcal{K}(\mathrm{m}, z) * \mathrm{f}(z)=z+\sum_{n=2}^{\infty} \frac{\mathrm{m}^{\mathrm{n}-1}}{(\mathrm{n}-1) !} e^{-\mathrm{m}} \mathrm{a}_{\mathrm{n}} z^{\mathrm{n}}, \quad z \in \mathcal{U},
$$


where $*$ denote the convolution or Hadamard product of two series.

Motivated by several earlier results on connections between various subclasses of analytic and univalent functions by using hypergeometric functions (see $[1,4,13,14])$ and by the recent investigations of Porwal $([10,12,11])$, in the present paper we determine the necessary and sufficient conditions for $\mathcal{F}(\mathrm{m}, z)$ to be in our new classes $\mathcal{S}(k, \lambda)$ and $\mathcal{C}(k, \lambda)$ and connections of these subclasses with $\mathcal{R}^{\tau}(A, B)$. Finally, we give conditions for the integral operator $\mathcal{G}(\mathrm{m}, z)=\int_{0}^{z} \frac{\mathcal{F}(\mathrm{m}, \mathrm{t})}{\mathrm{t}} \mathrm{dt}$ to be in the classes $\mathcal{S}(\mathrm{k}, \lambda)$ and $\mathcal{C}(k, \lambda)$.

To establish our main results, we will require the following Lemmas.

Lemma 1 [3] A function $\mathrm{f}$ of the form $(2)$ is in $\mathcal{S}(\mathrm{k}, \lambda)$ if and only if it satisfies

$$
\sum_{n=2}^{\infty}[n((1-\lambda)+k(1+\lambda))-(1-\lambda)(1-k)]\left|a_{n}\right| \leq 2 k
$$

where $0<k \leq 1$ and $0 \leq \lambda<1$. The result is sharp.

Lemma 2 [3] A function $\mathrm{f}$ of the form $(2)$ is in $\mathcal{C}(\mathrm{k}, \lambda)$ if and only if it satisfies

$$
\sum_{n=2}^{\infty} n[n((1-\lambda)+k(1+\lambda))-(1-\lambda)(1-k)]\left|a_{n}\right| \leq 2 k
$$

where $0<\mathrm{k} \leq 1$ and $0 \leq \lambda<1$. The result is sharp.

Lemma 3 [2] If $\mathrm{f} \in \mathcal{R}^{\tau}(\mathrm{A}, \mathrm{B})$ is of the form, then

$$
\left|a_{n}\right| \leq(A-B) \frac{|\tau|}{n}, \quad n \in \mathbb{N}-\{1\} .
$$

The result is sharp.

\section{The necessary and sufficient conditions}

Theorem 1 If $\mathrm{m}>0,0<\mathrm{k} \leq 1$ and $0 \leq \lambda<1$, then $\mathcal{F}(\mathrm{m}, z)$ is in $\mathcal{S}(\mathrm{k}, \lambda)$ if and only if

$$
((1-\lambda)+k(1+\lambda)) m e^{m} \leq 2 k .
$$

Proof. Since

$$
\mathcal{F}(m, z)=z-\sum_{n=2}^{\infty} \frac{m^{n-1}}{(n-1) !} e^{-m} z^{n}
$$


according to (3) of Lemma 1, we must show that

$$
\sum_{n=2}^{\infty}[n((1-\lambda)+k(1+\lambda))+(1-\lambda)(k-1)] \frac{m^{n-1}}{(n-1) !} \leq 2 k e^{m} .
$$

Writing $n=(n-1)+1$, we have

$$
\begin{aligned}
& \sum_{n=2}^{\infty}[n((1-\lambda)+k(1+\lambda))+(1-\lambda)(k-1)] \frac{m^{n-1}}{(n-1) !} \\
& \quad=\sum_{n=2}^{\infty}[(n-1)((1-\lambda)+k(1+\lambda))+2 k] \frac{m^{n-1}}{(n-1) !} \\
& \quad=[(1-\lambda)+k(1+\lambda)] \sum_{n=2}^{\infty} \frac{m^{n-1}}{(n-2) !}+2 k \sum_{n=2}^{\infty} \frac{m^{n-1}}{(n-1) !} \\
& \quad=((1-\lambda)+k(1+\lambda)) m e^{m}+2 k\left(e^{m}-1\right) .
\end{aligned}
$$

But this last expression is bounded above by $2 \mathrm{ke}^{\mathrm{m}}$ if and only if (5) holds.

Theorem 2 If $\mathrm{m}>0,0<\mathrm{k} \leq 1$ and $0 \leq \lambda<1$, then $\mathcal{F}(\mathrm{m}, z)$ is in $\mathcal{C}(k, \lambda)$ if and only if

$$
((1-\lambda)+k(1+\lambda)) m^{2} e^{m}+2(1+2 k+k \lambda-\lambda) m e^{m} \leq 2 k .
$$

Proof. In view of Lemma 2, it suffices to show that

$$
\sum_{n=2}^{\infty} n[n((1-\lambda)+k(1+\lambda))+(1-\lambda)(k-1)] \frac{m^{n-1}}{(n-1) !} \leq 2 k e^{m} .
$$

Now

$$
\begin{aligned}
\sum_{n=2}^{\infty} n & {[n((1-\lambda)+k(1+\lambda))+(1-\lambda)(k-1)] \frac{m^{n-1}}{(n-1) !} } \\
= & \left.\sum_{n=2}^{\infty} n^{2}((1-\lambda)+k(1+\lambda))+n(1-\lambda)(k-1)\right] \frac{m^{n-1}}{(n-1) !} .
\end{aligned}
$$

Writing $n=(n-1)+1$ and $n^{2}=(n-1)(n-2)+3(n-1)+1$, in (10) we see that

$$
\left.\sum_{n=2}^{\infty} n^{2}((1-\lambda)+k(1+\lambda))+n(1-\lambda)(k-1)\right] \frac{m^{n-1}}{(n-1) !}
$$




$$
\begin{aligned}
= & \sum_{n=2}^{\infty}(n-1)(n-2)((1-\lambda)+k(1+\lambda)) \frac{m^{n-1}}{(n-1) !} \\
& +\sum_{n=2}^{\infty}(n-1)\left[3((1-\lambda)+k(1+\lambda)+(1-\lambda)(k-1)] \frac{m^{n-1}}{(n-1) !}+\sum_{n=2}^{\infty} 2 k \frac{m^{n-1}}{(n-1) !}\right. \\
= & ((1-\lambda)+k(1+\lambda)) \sum_{n=2}^{\infty} \frac{m^{n-1}}{(n-3) !}+2(1+2 k+k \lambda-\lambda) \sum_{n=2}^{\infty} \frac{m^{n-1}}{(n-2) !} \\
& +2 k \sum_{n=2}^{\infty} \frac{m^{n-1}}{(n-1) !} \\
= & ((1-\lambda)+k(1+\lambda)) m^{2} e^{m}+2(1+2 k+k \lambda-\lambda) m e^{m}+2 k\left(e^{m}-1\right) .
\end{aligned}
$$

But this last expression is bounded above by $2 \mathrm{ke}^{\mathrm{m}}$ if and only if (9) holds.

By specializing the parameter $\lambda=0$ in Theorems 1 and 2 , we have the following corollaries.

Corollary 1 If $\mathrm{m}>0$ and $0<\mathrm{k} \leq 1$, then $\mathcal{F}(\mathrm{m}, z)$ is in $\mathcal{S}(\mathrm{k})$ if and only if

$$
(1+k) m e^{m} \leq 2 k .
$$

Corollary 2 If $\mathrm{m}>0$ and $0<\mathrm{k} \leq 1$, then $\mathcal{F}(\mathrm{m}, z)$ is in $\mathcal{C}(\mathrm{k})$ if and only if

$$
(1+k) m^{2} e^{m}+2(1+2 k) m e^{m} \leq 2 k .
$$

\section{Inclusion properties}

Theorem 3 Let $\mathrm{m}>0,0<\mathrm{k} \leq 1$ and $0 \leq \lambda<1$. If $\mathrm{f} \in \mathcal{R}^{\tau}(\mathrm{A}, \mathrm{B})$, then $\mathcal{I}(\mathrm{m}, z) \mathrm{f}$ is in $\mathcal{S}(\mathrm{k}, \lambda)$ if and only if

$$
\begin{aligned}
& (A-B)|\tau|\left[((1-\lambda)+k(1+\lambda))\left(1-e^{-m}\right)\right. \\
& \left.+\frac{(1-\lambda)(k-1)}{m}\left(1-e^{-m}(1+m)\right)\right] \leq 2 k .
\end{aligned}
$$

Proof. In view of Lemma 1, it suffices to show that

$$
\sum_{n=2}^{\infty}[n((1-\lambda)+k(1+\lambda))+(1-\lambda)(k-1)] \frac{m^{n-1}}{(n-1) !}\left|a_{n}\right| \leq 2 k e^{m} .
$$

Since $f \in \mathcal{R}^{\tau}(A, B)$, then by Lemma 3 , we get

$$
\left|a_{n}\right| \leq \frac{(A-B)|\tau|}{n}
$$


Thus, we have

$$
\begin{aligned}
& \sum_{n=2}^{\infty}[n((1-\lambda)+k(1+\lambda))+(1-\lambda)(k-1)] \frac{m^{n-1}}{(n-1) !}\left|a_{n}\right| \\
& \quad \leq(A-B)|\tau| \sum_{n=2}^{\infty}[n((1-\lambda)+k(1+\lambda))+(1-\lambda)(k-1)] \frac{m^{n-1}}{n !} \\
& \quad=(A-B)|\tau|\left[((1-\lambda)+k(1+\lambda)) \sum_{n=2}^{\infty} \frac{m^{n-1}}{(n-1) !}+\frac{(1-\lambda)(k-1)}{m} \sum_{n=2}^{\infty} \frac{m^{n}}{n !}\right] \\
& \quad=(A-B)|\tau|\left[((1-\lambda)+k(1+\lambda))\left(e^{m}-1\right)+\frac{(1-\lambda)(k-1)}{m}\left(e^{m}-1-m\right)\right] .
\end{aligned}
$$

But this last expression is bounded above by $2 \mathrm{ke}^{\mathrm{m}}$ if and only if (13) holds.

Theorem 4 Let $\mathrm{m}>0,0<\mathrm{k} \leq 1$ and $0 \leq \lambda<1$. If $\mathrm{f} \in \mathcal{R}^{\tau}(\mathrm{A}, \mathrm{B})$, then $\mathcal{F}(\mathrm{m}, z) \mathrm{f}$ is in $\mathcal{C}(\mathrm{k}, \lambda)$ if and only if

$$
(A-B)|\tau|\left[((1-\lambda)+k(1+\lambda)) m+2 k\left(1-e^{-m}\right)\right] \leq 2 k .
$$

Proof. In view of Lemma 2, it suffices to show that

$$
\sum_{n=2}^{\infty} n[n((1-\lambda)+k(1+\lambda))+(1-\lambda)(k-1)] \frac{m^{n-1}}{(n-1) !}\left|a_{n}\right| \leq 2 k e^{m} .
$$

Using (14), we have

$$
\begin{aligned}
& \sum_{n=2}^{\infty} n[n((1-\lambda)+k(1+\lambda))+(1-\lambda)(k-1)] \frac{m^{n-1}}{(n-1) !}\left|a_{n}\right| \\
& \quad \leq \sum_{n=2}^{\infty} n[n((1-\lambda)+k(1+\lambda))+(1-\lambda)(k-1)] \frac{m^{n-1}}{(n-1) !} \frac{(A-B)|\tau|}{n} \\
& \quad=(A-B)|\tau| \sum_{n=2}^{\infty}[n((1-\lambda)+k(1+\lambda))+(1-\lambda)(k-1)] \frac{m^{n-1}}{(n-1) !} \\
& \quad=(A-B)|\tau| \sum_{n=2}^{\infty}[(n-1)((1-\lambda)+k(1+\lambda))+2 k] \frac{m^{n-1}}{(n-1) !} \\
& \quad=(A-B)|\tau|\left[((1-\lambda)+k(1+\lambda)) \sum_{n=2}^{\infty} \frac{m^{n-1}}{(n-2) !}+2 k \sum_{n=2}^{\infty} \frac{m^{n-1}}{(n-1) !}\right]
\end{aligned}
$$




$$
=(A-B)|\tau|\left[((1-\lambda)+k(1+\lambda)) m e^{m}+2 k\left(e^{m}-1\right)\right] .
$$

But this last expression is bounded above by $2 \mathrm{ke}^{\mathrm{m}}$ if and only if (15) holds.

By taking $\lambda=0$ in Theorems 3 and 4, we obtain the following corollaries.

Corollary 3 Let $\mathrm{m}>0$ and $0<\mathrm{k} \leq 1$. If $\mathrm{f} \in \mathcal{R}^{\tau}(\mathrm{A}, \mathrm{B})$, then $\mathcal{I}(\mathrm{m}, z) \mathrm{f}$ is in $\mathcal{S}(\mathrm{k})$ if and only if

$$
(A-B)|\tau|\left[(1+k)\left(1-e^{-m}\right)+\frac{(k-1)}{m}\left(1-e^{-m}(1+m)\right)\right] \leq 2 k .
$$

Corollary 4 Let $\mathrm{m}>0$ and $0<\mathrm{k} \leq 1$. If $\mathrm{f} \in \mathcal{R}^{\tau}(\mathrm{A}, \mathrm{B})$, then $\mathcal{I}(\mathrm{m}, z) \mathrm{f}$ is in $\mathcal{C}(\mathrm{k})$ if and only if

$$
(A-B)|\tau|\left[(1+k) m+2 k\left(1-e^{-m}\right)\right] \leq 2 k .
$$

\section{An integral operator}

In this section, we obtain the necessary and sufficient conditions for the integral operator $\mathcal{G}(m, z)$ defined by

$$
\mathcal{G}(\mathrm{m}, z)=\int_{0}^{z} \frac{\mathcal{F}(\mathrm{m}, \mathrm{t})}{\mathrm{t}} \mathrm{dt}
$$

to be in the class $\mathcal{C}(k, \lambda)$.

Theorem 5 If $\mathrm{m}>0,0<\mathrm{k} \leq 1$ and $0 \leq \lambda<1$, then the integral operator $\mathcal{G}(\mathrm{m}, z)$ defined by $(18)$ is in $\mathcal{C}(\mathrm{k}, \lambda)$ if and only if (5) is satisfied.

Proof. Since

$$
\mathcal{G}(\mathrm{m}, z)=z-\sum_{\mathrm{n}=2}^{\infty} \frac{\mathrm{e}^{-\mathrm{m}} \mathrm{m}^{\mathrm{n}-1}}{\mathrm{n} !} z^{\mathrm{n}}
$$

then by Lemma 2, we need only to show that

$$
\sum_{n=2}^{\infty} n[n((1-\lambda)+k(1+\lambda))+(1-\lambda)(k-1)] \frac{m^{n-1}}{n !} \leq 2 k e^{m} .
$$

or, equivalently

$$
\sum_{n=2}^{\infty}[n((1-\lambda)+k(1+\lambda))+(1-\lambda)(k-1)] \frac{m^{n-1}}{(n-1) !} \leq 2 k e^{m}
$$


From (8) it follows that

$$
\begin{aligned}
& \sum_{n=2}^{\infty}[n((1-\lambda)+k(1+\lambda))+(1-\lambda)(k-1)] \frac{m^{n-1}}{(n-1) !} \\
& \quad=((1-\lambda)+k(1+\lambda)) m e^{m}+2 k\left(e^{m}-1\right)
\end{aligned}
$$

and this last expression is bounded above by $2 k e^{m}$ if and only if (5) holds.

The proof of Theorem 6 (below) is much similar to that of Theorem 5 and so the details are omitted.

Theorem 6 If $\mathrm{m}>0,0<\mathrm{k} \leq 1$ and $0 \leq \lambda<1$, then the integral operator $\mathcal{G}(\mathrm{m}, z)$ defined by $(18)$ is in $\mathcal{S}(\mathrm{k}, \lambda)$ if and only if

$$
((1-\lambda)+k(1+\lambda))\left(1-e^{-m}\right)+\frac{(1-\lambda)(k-1)}{m}\left(1-e^{-m}-m e^{-m}\right) \leq 2 k .
$$

By taking $\lambda=0$ in Theorems 5 and 6 , we obtain the following corollaries.

Corollary 5 If $\mathrm{m}>0$ and $0<\mathrm{k} \leq 1$, then the integral operator defined by (18) is in $\mathcal{C}(\mathrm{k})$ if and only if (11) is satisfied.

Corollary 6 If $\mathrm{m}>0$ and $0<\mathrm{k} \leq 1$, then the integral operator defined by (18) is in $\mathcal{S}(\mathrm{k})$ if and only if

$$
(1+k)\left(1-e^{-m}\right)+\frac{(k-1)}{m}\left(1-e^{-m}-m e^{-m}\right) \leq 2 k .
$$

\section{Acknowledgements}

The author would like to thank the referee for his helpful comments and suggestions.

\section{References}

[1] N. E. Cho, S. Y. Woo, S. Owa, Uniform convexity properties for hypergeometric functions, Fract. Cal. Appl. Anal., 5 (3) (2002), 303-313.

[2] K. K. Dixit, S. K. Pal, On a class of univalent functions related to complex order, Indian J. Pure Appl. Math., 26 (9) (1995), 889-896. 
[3] B. A. Frasin, T. Al-Hawary, F. Yousef, Necessary and sufficient conditions for hypergeometric functions to be in a subclass of analytic functions, Afr. Mat., 30 (1-2) (2019), 223-230.

[4] E. Merkes, B. T. Scott, Starlike hypergeometric functions, Proc. Amer. Math. Soc., 12 (1961), 885-888.

[5] M. L. Mogra, On a class of starlike functions in the unit disc I, J. Indian Math. Soc. 40 (1976), 159-161.

[6] G. Murugusundaramoorthy, Subclasses of starlike and convex functions involving Poisson distribution series, Afr. Mat. (2017) 28:1357-1366.

[7] G. Murugusundaramoorthy, K. Vijaya, S. Porwal, Some inclusion results of certain subclass of analytic functions associated with Poisson distribution series, Hacettepe J. Math. Stat., 45 (4) (2016), 1101-1107.

[8] S. Owa, On certain classes of univalent functions in the unit disc, Kyungpook Math. J., 24 (2) (1984), 127-136.

[9] K. S. Padmanabhan, On certain classes of starlike functions in the unit disc, J. Indian Math. Soc., 32 (1968), 89-103.

[10] S. Porwal, An application of a Poisson distribution series on certain analytic functions, J. Complex Anal., (2014), Art. ID 984135, 1-3.

[11] S. Porwal, Mapping properties of generalized Bessel functions on some subclasses of univalent functions, Anal. Univ. Oradea Fasc. Matematica, 20 (2) (2013), 51-60.

[12] S. Porwal, M. Kumar, A unified study on starlike and convex functions associated with Poisson distribution series, Afr. Mat., 27 (5) (2016), 10211027.

[13] H. Silverman, Starlike and convexity properties for hypergeometric functions, J. Math. Anal. Appl., 172 (1993), 574-581.

[14] H. M. Srivastava, G. Murugusundaramoorthy, S. Sivasubramanian, Hypergeometric functions in the parabolic starlike and uniformly convex domains, Integr. Transf. Spec. Func., 18 (2007), 511-520.101-1107. 